\title{
Design of a Compact and High Sensitivity Temperature Sensor Using Metamaterial
}

\author{
Sabah Zemouli, ${ }^{1}$ Abdelhafid Chaabi, ${ }^{1}$ and Houcine Sofiane Talbi ${ }^{2}$ \\ ${ }^{1}$ Laboratoire Hyperfréquence et Semiconducteur Université Constantine 1, BP 325, Route Ain El Bey, 25017 Constantine, Algeria \\ ${ }^{2}$ Faculté de Médecine, Université de Batna, 05 avenue Chahid Boukhlouf, 05000 Batna, Algeria \\ Correspondence should be addressed to Sabah Zemouli; sabahzemouli@gmail.com
}

Received 9 October 2014; Revised 15 December 2014; Accepted 8 January 2015

Academic Editor: Yun-Gui Ma

Copyright (c) 2015 Sabah Zemouli et al. This is an open access article distributed under the Creative Commons Attribution License, which permits unrestricted use, distribution, and reproduction in any medium, provided the original work is properly cited.

\begin{abstract}
The present paper aims at sensing the temperature. A sensor metamaterial consisting of two concentric metallic rings and a thin metallic wire deposited on the surface of $\mathrm{BaTiO} 3$ substrate is reported. The use of $\mathrm{BaTiO} 3$ makes the resonant frequency of the structure shift as the temperature varies and makes the sensor applicable in many fields of applications. Numerical simulations and theoretical results are presented and compared to each other; there was a good agreement between them. This sensor is smaller, easier to fabricate, and very sensitive to the changes in temperatures.
\end{abstract}

\section{Introduction}

In this decade, metamaterials (MTM) or left handed materials (LHM) have attracted much attention among researchers in the microwave communities because of their unusual properties, such as negative permittivity [1], negative permeability [2], and negative refractive index [3,4]. These periodic materials are very useful for many fields of applications [5-7]. Recently, a great interest has been devoted to the application of metamaterials in sensing; hereafter, we have provided some researches in this concern. Ishimaru and his colleagues have studied a metamaterial based on surface plasmon resonance (SPR) sensors in $\mathrm{GHz}$ regime while the traditional SPR is usually operated in optical domain only [8]. Kabashin et al. in [9] have enhanced the SPR sensor's performances by using metamaterial. In [10], the authors have proposed a method of dielectric sensing using $\varepsilon$ near zero narrow waveguide channels. In [11], Ekmekci and his colleague have studied a double sided square SRR (split ring resonator) topology for multipurpose sensor operating in $X$ band; this sensor exhibits a negative value of permeability for frequencies close to the magnetic resonance frequency of the SRR structure, whereas our suggested structure exhibits negative permittivity and negative permeability close to the magnetic resonance frequency of the structure; in addition and according to [12] circular SRR can present a higher quality factor compared to square SRR. References $[13,14]$ have studied the application of a Terahertz metamaterial for chemical sensing and [15] have explained how metamaterials can offer solutions to overcome technical limitations of wireless sensors.

Metamaterials are better in sensing than the conventional resonators (e.g., spiral coils), because they are easy and rapid in detection [16-18] and they can exhibit a strong localization and enhancement of fields; that is, they can improve the sensor's selectivity and sensibility [19] due to the application of an axial time varying magnetic field parallel to the particle plane in the orthogonal direction to the splits which creates a huge electric field density localized between them and presents higher signal and higher dips in their transmission spectrum, which results in a high quality factor $Q$ compared to the conventional RF (radio frequency) resonators. This enables us to make telemetric measurements and to observe the operating frequency relatively easier [20]. Another advantage of the gaps existence in the MTM structure is the production of an additional capacitive effect which exhibits a lower resonance frequency (hence avoid the background absorption of the structure) and results in higher shift of the operating frequency with the same applied load, leading to higher sensitivity. The use of Barium titanate dielectric that is very sensitive to the changes of temperature makes our sensor more sensitive. 
The aim of this paper is to design a high sensitive and smaller electrical sensor of temperature based on metamaterial operating in $\mathrm{GHz}$ regime. The proposed structure consists of a combination of two concentric circular metallic rings opened in the opposite sides commonly called SRR and a thin metallic wire placed on a dielectric substrate. Because the resonant frequency of the MTM structure is very sensitive to the changes of the capacitive effects (since its fundamental resonance behavior can be modeled by an LC circuit [21]) which are basically dependent on the resonator's topology and the substrate's parameters (i.e., substrate's thickness and relative permittivity), we have proposed a structure with a varying dielectric characteristics substrate. When the temperature changes, the dielectric properties of the substrate of the MTM will change and this will affect the capacitance of the SRR and then a shift in the resonant frequency will appear; this will indicate the measured temperature.

In this work, we have designed the equivalent circuit model of the proposed structure and we have derived the relations of its coefficients of reflexion and transmission using the ABCD Matrix approach; then we have compared them to those resulting from the simulation. The results were very close. In this study the metamaterial sensor is smaller, is easier to fabricate, possesses higher sensitivity, and is useful in a wide range of applications. The mechanism behind this is the use of a varying dielectric characteristics substrate.

\section{Design OF LHM}

The MTM structure is composed of a combination of PEC (perfect electric conductor) circular SRR and thin wire placed on a dielectric substrate of $0.1 \mathrm{~mm}$ of thickness $\left(t_{d}\right)$; the length and width of this substrate are $2 \mathrm{~mm}$ and $2.5 \mathrm{~mm}$, respectively. The external radius of the SRR is $r_{0}=0.75 \mathrm{~mm}$, the width of the two rings is $d=0.18 \mathrm{~mm}$, the spacing between them is $0.24 \mathrm{~mm}$, the split of the outer ring is $0.4 \mathrm{~mm}$, the split of the inner ring is equal to $0.2 \mathrm{~mm}$, and the thickness of the rings is $t_{m}=0.035 \mathrm{~mm}$; the wire has a $0.2 \mathrm{~mm}$ of width and $1.8 \mathrm{~mm}$ of length. The substrate of this model is the Barium titanate dielectric BaTiO3 $[22,23]$ which is one of the best known and commonly used materials for electric ceramics due to its excellent dielectric, ferroelectric, and piezoelectric properties at and above room temperature. We have specially chosen this material because its relative permittivity has a big dependence with the temperature (see Figure 1) which can be tailored in a wide range of applications. Furthermore, this material is sintered at high temperatures. This allows an operation at very elevated temperatures. Figure 2 shows the proposed structure.

\section{Simulation and Equivalent Circuit Model}

The simulation of the MTM structure has been executed using the High Frequency Structure Simulator (HFSS) software. A perfect magnetic conductor (PMC) boundary condition was set along $z$-axis while the perfect electric conductor (PEC) boundary condition was set along $y$-axis. On the last two faces, we have set two ports rectangular waveguide for sensor measurements. The LHM structure was placed at the center of the waveguide.

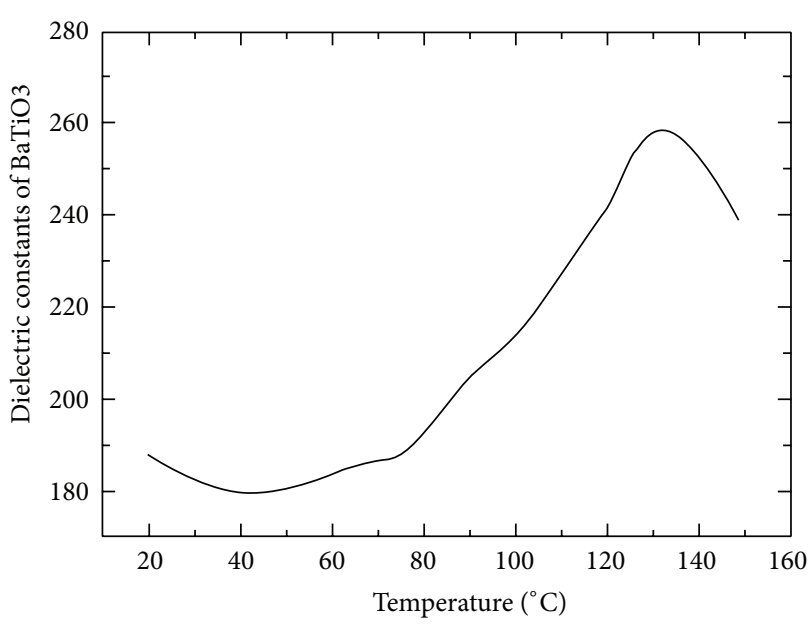

Figure 1: Variation of the dielectric constant of $\mathrm{BaTiO} 3$ versus the temperature [22].

In Figure 3, we have studied the variation of the coefficient of the transmission S21 versus the variation of the frequency using the commercial simulator HFSS to study the shift in the resonant frequency according to the variation of the temperature of the sensor.

The effective permittivity of the LHM structure is different from the relative permittivity of the $\mathrm{BaTiO} 3$ substrate $\left(\varepsilon_{\mathrm{BaTiO} 3} \neq \varepsilon_{\mathrm{MTM} \text { structure }}\right)$. We have calculated the effective permittivity $\varepsilon_{\text {eff }}$ and the effective permeability $\mu_{\text {eff }}$ of the MTM structure using the Nicolson-Ross-Weir method [24]; the results are shown in Figure 4.

We notice that the resonant frequency of the simulated structure is $2.3 \mathrm{GHz}$ with return loss of $-20 \mathrm{~dB}$. The relative permittivity at this frequency is -12.19 , whereas the relative permeability is -12.65 .

3.1. Study of the Effects of the Dielectric Substrate on the MTM Structure. The resonant frequency of the MTM structure is inversely related to the total capacitance $C_{T}$ of the sensor $f=1 / 2 \pi \sqrt{L_{T} C_{T}}[21]$; it is known that the capacitance is proportional to the dielectric constant $C=\varepsilon(l / S)$, where $S$ is area of a dielectric material, $l$ is the thickness of a dielectric material, and $\varepsilon$ is dielectric material's permittivity.

When $\varepsilon$ increases, the capacitance of the sensor $C_{T}$ increases which will decrease the resonant frequency. The shift in the resonant frequency resulting from the variation of the permittivity will indicate the variation in the temperature since the dielectric characteristics of the $\mathrm{BaTiO} 3$ substrate are related to the temperature (see Figure 1).

When a time harmonic external magnetic field is applied along $z$ axis of this structure, an electromotive force will create an induced current in the structure that will explain the inductive part of our system.

Because the LHM structure has a size much smaller than the operating wavelength, we can describe its electromagnetic characteristics by lumped circuit components capacitors and inductors. The overall structure can be represented by an $L C$ circuit and the equivalent circuit model proposed for our 


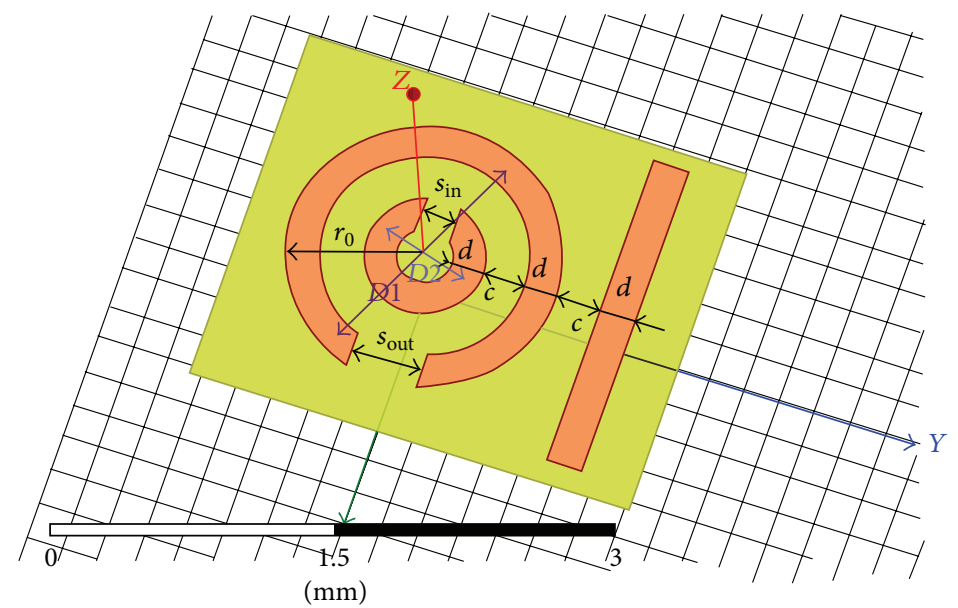

(a)

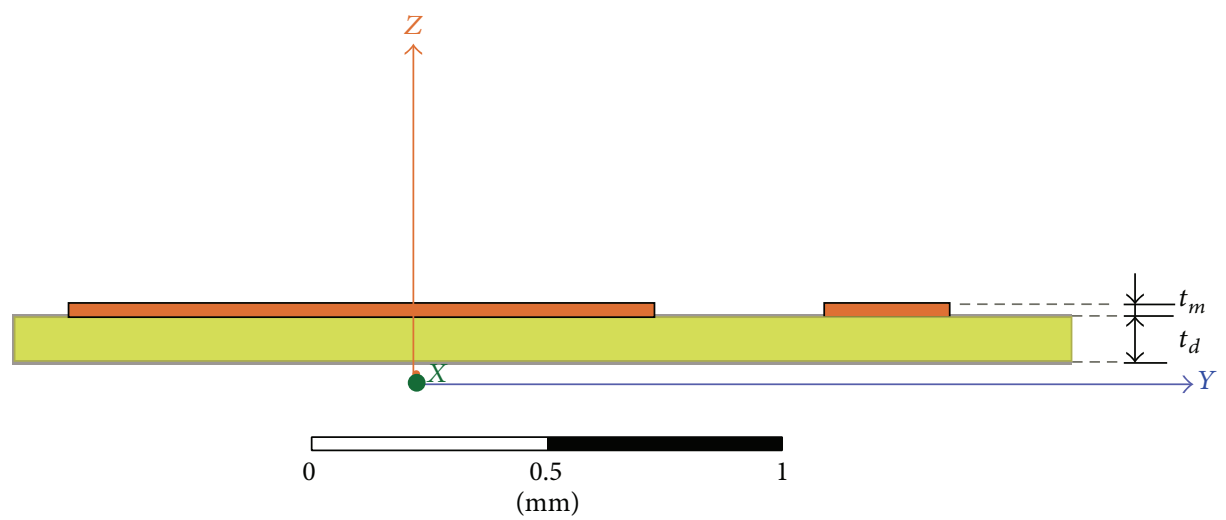

(b)

FIGURE 2: SRR $r_{0}=0.75 \mathrm{~mm}$, width $d=0.18 \mathrm{~mm}$, spacing between the two rings $c=0.24 \mathrm{~mm}$, thickness of the SRR $t_{m}=0.035 \mathrm{~mm}$, and the split of the outer ring $s_{\text {out }}=0.4 \mathrm{~mm}$ and of the inner ring $s_{\text {in }}=0.2 \mathrm{~mm}$; the wire has $0.2 \mathrm{~mm}$ of width and $1.8 \mathrm{~mm}$ of length.

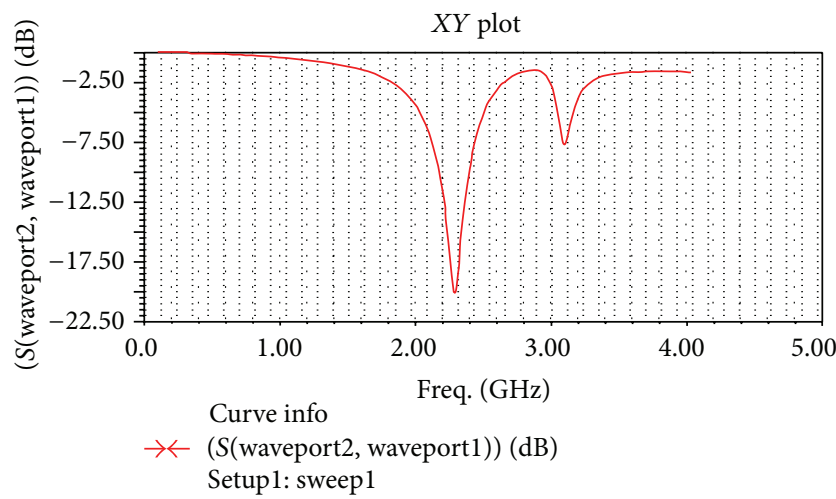

FIGURE 3: Variation of the coefficient of transmission S21 versus frequency at $40^{\circ} \mathrm{C}$.

LHM structure is shown in Figure 6, where $L$ models the total inductance of the two rings of the SRR, $C$ is the capacitance of the split in the inner and outer ring and the capacitance of the gap between them, $C_{s}$ models the coupling between the SRR and the line, and $L_{s}$ models the inductance of the wire. After calculation, we noticed $L \ggg M$, so the mutual inductance can be neglected and the inductive currents will be neglected.

Figure 5 shows the distribution of the current around the wire and the SRR and demonstrates the previous conclusion.

In Figure 7, we have plotted the calculated S21 parameter versus the frequency using ABCD Matrix approach, which is based on the following equations:

$$
\begin{gathered}
A=\left.\frac{V_{1}}{V_{2}}\right|_{I 2=0}, \quad B=\left.\frac{V_{1}}{I_{2}}\right|_{V 2=0}, \\
C=\left.\frac{I_{1}}{V_{2}}\right|_{I 2=0}, \quad D=\left.\frac{I_{1}}{I_{2}}\right|_{V 2=0}, \\
Z_{11}=\frac{A}{C}, \quad Z_{12}=\frac{(A D-B C)}{C}, \\
Z_{21}=\frac{1}{C}, \quad Z_{22}=\frac{D}{C}, \\
S 11=\frac{\left[\left(Z_{11}-Z_{0}\right)\left(Z_{22}+Z_{0}\right)-Z_{12} Z_{21}\right]}{\Delta Z},
\end{gathered}
$$




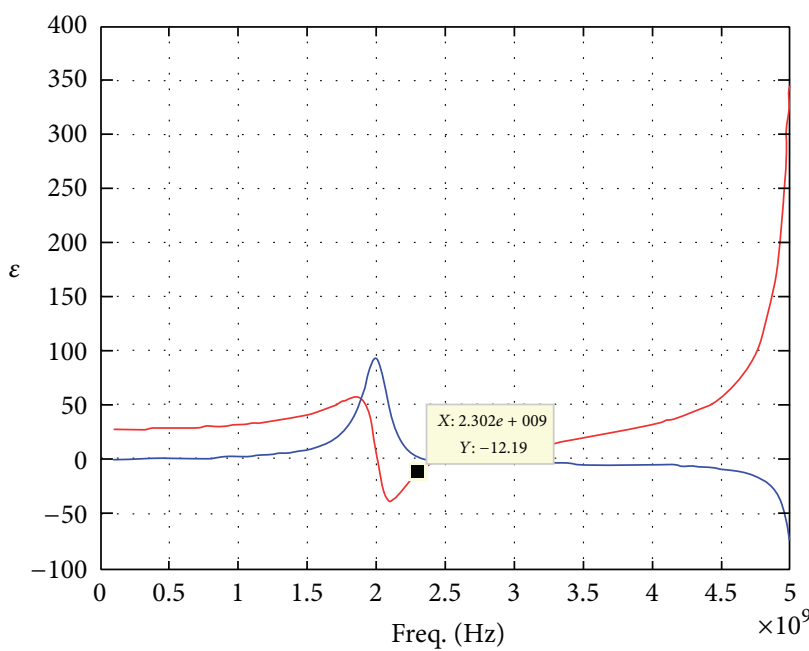

(a)

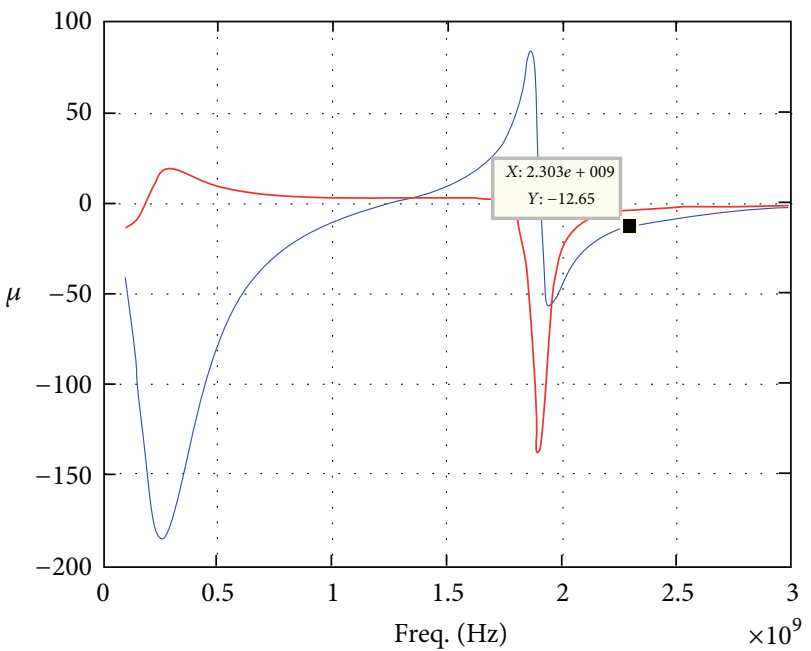

(b)

FIGURE 4: The variation of the real (red) and imaginary part (blue) of the permittivity $(\varepsilon)$ and the variation of the real (red) and imaginary part (blue) of the permeability $(\mu)$ according to the frequency.

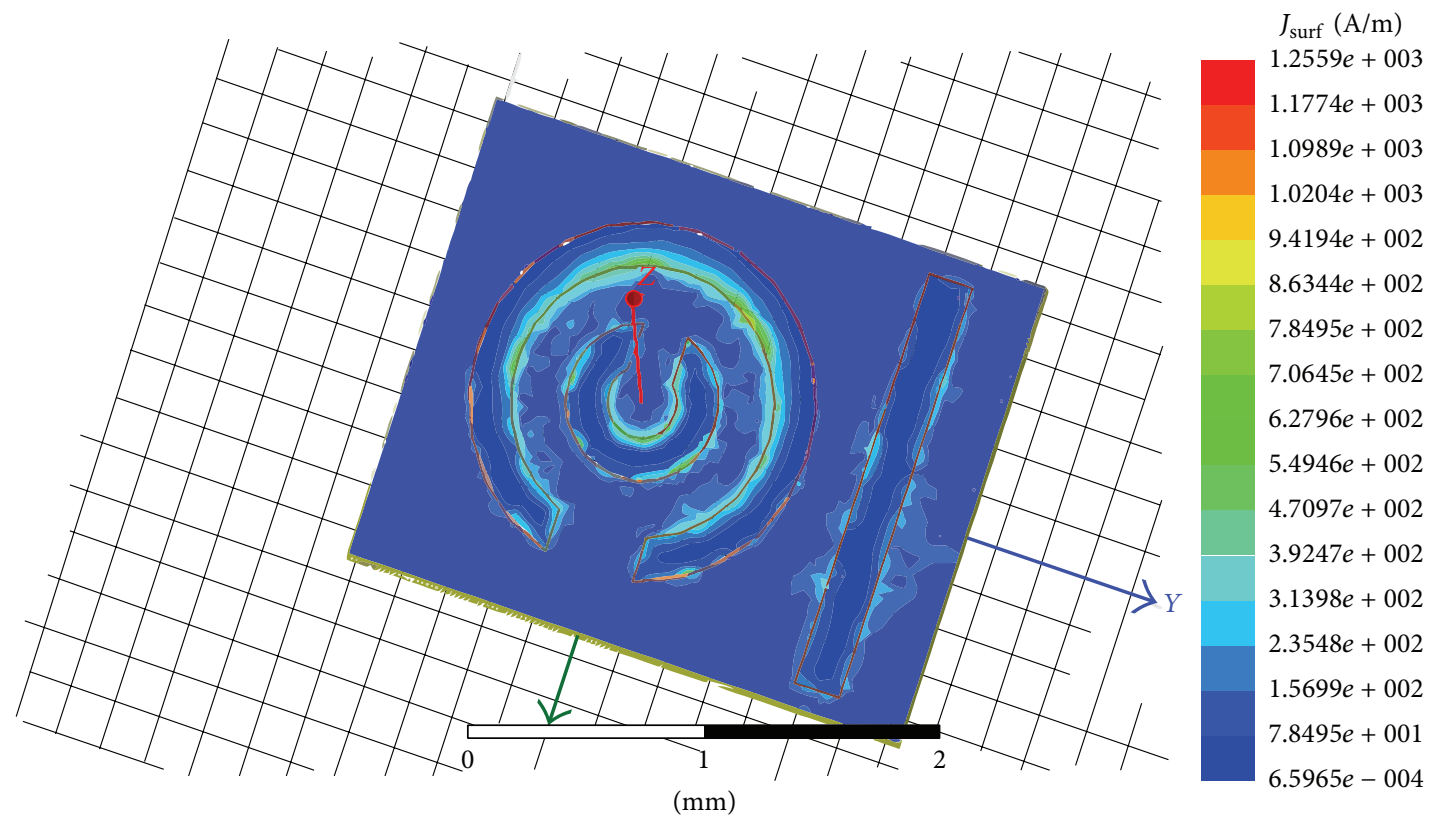

FIgURE 5: The distribution of the current around the wire and the SRR.

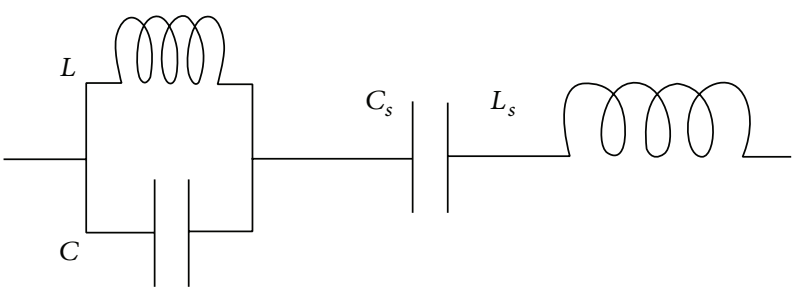

FIGURE 6: Equivalent circuit model for the LHM structure. 


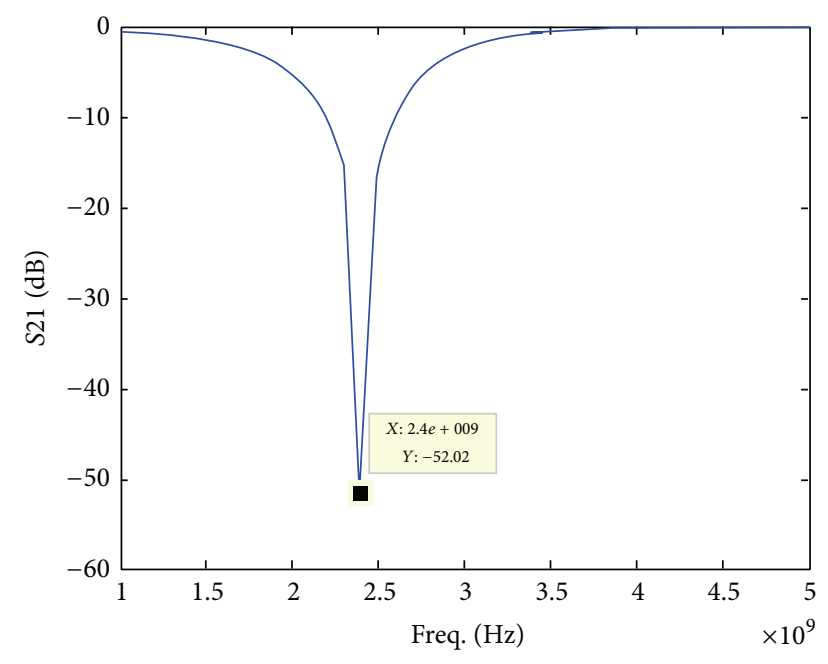

FIGURE 7: The variation of S21 versus frequency calculated using the ABCD Matrix approach from the equivalent circuit model.

$$
\begin{gathered}
S 21=\frac{\left[\left(Z_{11}+Z_{0}\right)\left(Z_{22}-Z_{0}\right)-Z_{12} Z_{21}\right]}{\Delta Z}, \\
\Delta Z=\left(Z_{11}+Z_{0}\right)\left(Z_{22}+Z_{0}\right)-Z_{12} Z_{21} .
\end{gathered}
$$

The ABCD Matrix for this kind of a cascade connection of two port networks is $\left[\begin{array}{cc}1 & Z_{T} \\ 0 & 1\end{array}\right]$ with $Z_{T}=j \omega L_{s}+1 / j \omega C_{s}+$ $\left(1-L C \omega^{2}\right) / j C \omega$; the parameters $L, C, L_{s}$, and $C_{s}$ can be approximated by the following relationships $[25,26]$ :

$$
\begin{gathered}
L=8 \mu D\left[\ln \left(\frac{D}{d}\right)+0.9+0.2 \frac{\omega^{2}}{D^{2}}+\frac{0.5 c^{2}}{(\rho D)^{2}}\right. \\
\left.+\frac{0.4 s(c+d)}{D^{2}}-0.5 \ln \left(\frac{d+t_{m}}{d}\right)\right] \\
+2 \mu D\left[\ln \left(\frac{1}{\rho}\right)-0.6+0.7 \rho^{2}+\left(0.2+\frac{1}{12 \rho^{2}}\right) \frac{d^{2}}{D^{2}}\right], \\
C=\left[0.06+3.5 \times 10^{-5} \times\left(r_{\text {out }}+r_{\text {in }}\right)\right]+\varepsilon \varepsilon_{0} \frac{d t_{m}}{s} \\
L_{s}=\frac{\mu l}{\pi}\left[2 \ln \left(\frac{2.067 l}{\omega}\right)+0.36 \frac{\omega}{l}+0.25 \frac{\omega^{2}}{l^{2}}\right], \\
\quad C_{s}=\varepsilon \varepsilon_{0} \frac{d t_{m}}{c}, \\
\rho=\frac{d+c}{D}, \quad D=0.5(D 1+D 2) .
\end{gathered}
$$

The resonant frequency calculated is $2.4 \mathrm{GHz}$ with return loss of $-52 \mathrm{~dB}$. We note that the calculated and the simulated results are very close.

Each time the temperature varies, it leads to a variation in the dielectric characteristics of the substrate $\mathrm{BaTiO}_{3}$ of the

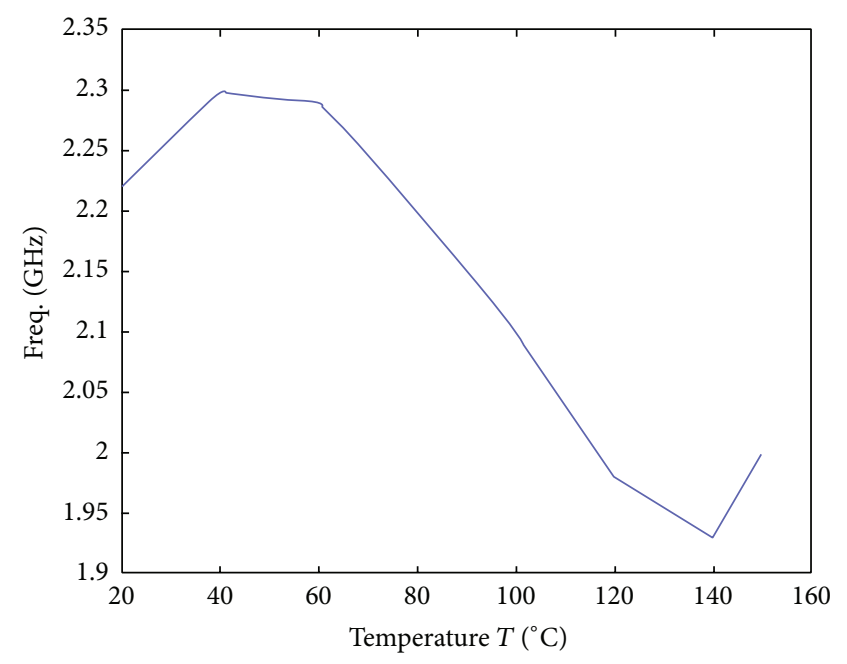

FIGURE 8: Variation of frequency versus the temperature.

LHM structure which affects its capacitance which depends inversely on the frequency of resonance of the structure.

To obtain the effects of the temperature on the $S$ parameters and hence on the resonant frequency of the MTM structure, we have studied the variation of the dielectric characteristics of the $\mathrm{BaTiO} 3$ that are corresponding to well-known temperatures according to the variation of the resonant frequency of the MTM structure. In the interval of temperature from $20^{\circ}$ to $150^{\circ}$, we have plotted the variation of the resonance frequency in function of temperature (see Figure 8).

The minimum and maximum temperatures that can be detected by this sensor depend on the minimum and maximum temperatures that can be supported by the $\mathrm{BaTiO} 3$ substrate.

The $\mathrm{BaTiO} 3$ material is sintered at high temperatures, which allows an operation at wide range temperatures [27]. So, TMax has a high value and because this material has no linear variation of the temperature in function of $\varepsilon$, the variation of the resonance frequency will not have a linear variation in function of the temperature (when $\varepsilon$ increases the resonant frequency of the MTM structure decreases; this is because the frequency is inversely proportional to the capacitive effect which is proportional to epsilon), but we can note that the curve is linear just in the intervals [20-40], [40-60], [60-120], and [120-140].

We have done this study in $\mathrm{GHz}$ regime, but the variations of the frequency are in the order of MHz; that is why in Table 1 we have presented the sensibility of this sensor in $\mathrm{MHz}$.

\section{Conclusion}

This paper presents a sensor of temperature using the metamaterial. We have studied the variations of the resonant frequency according to the permittivity of structure which depend on the temperature.

We have proposed an equivalent circuit model of our LHM structure and using the software MATLAB we have 
TABLE 1

\begin{tabular}{lcc}
\hline The interval of temperature & $\Delta f[\mathrm{MHz}]$ & $\begin{array}{c}\text { The } \\
\text { sensibility } \\
{[\mathrm{MHZ}]}\end{array}$ \\
\hline$\left[20^{\circ}-40^{\circ}\right]$ & 80 & 4 \\
{$\left[40^{\circ}-60^{\circ}\right]$} & 30 & 1.5 \\
{$\left[60^{\circ}-80^{\circ}\right]$} & 90 & 4.5 \\
{$\left[80^{\circ}-100^{\circ}\right]$} & 100 & 5 \\
{$\left[100^{\circ}-120^{\circ}\right]$} & 140 & 7 \\
{$\left[120^{\circ}-140^{\circ}\right]$} & 70 & 3.5 \\
\hline
\end{tabular}

calculated the coefficients of reflection and transmission; the obtained curves are in accordance with those obtained by the HFSS.

Using the two methods HFSS and Matrix ABCD, we can notice that the variation in the temperature involves a variation of the frequency of resonance and it is illustrated in a curve.

Because the $\mathrm{BaTiO} 3$ has big dependence of the relative permittivity with the temperature, this sensor can be tailored in a wide range of applications.

Based on the promising results of this sensor, we believe that the use of the proposed sensor's topology will efficiently be used in various sensing applications including pressure and humidity and also in biological and chemical sensing for any region of wavelengths.

\section{Conflict of Interests}

The authors declare that there is no conflict of interests regarding the publication of this paper.

\section{References}

[1] J. B. Pendry, A. J. Holden, W. J. Stewart, and I. Youngs, "Extremely low frequency plasmons in metallic mesostructures," Physical Review Letters, vol. 76, no. 25, pp. 4773-4776, 1996.

[2] J. B. Pendry, A. J. Holden, D. J. Robbins, and W. J. Stewart, "Magnetism from conductors and enhanced nonlinear phenomena," IEEE Transactions on Microwave Theory and Techniques, vol. 47, no. 11, pp. 2075-2084, 1999.

[3] D. R. Smith, W. J. Padilla, D. C. Vier, S. C. Nemat-Nasser, and S. Schultz, "Composite medium with simultaneously negative permeability and permittivity," Physical Review Letters, vol. 84, no. 18, pp. 4184-4187, 2000.

[4] R. A. Shelby, D. R. Smith, and S. Schultz, "Experimental verification of a negative index of refraction," Science, vol. 292, no. 5514, pp. 77-79, 2001.

[5] X. Shen, T. J. Cui, J. Zhao, H. F. Ma, W. X. Jiang, and H. Li, "Polarization-independent wide-angle triple-band metamaterial absorber," Optics Express, vol. 19, no. 10, pp. 9401-9407, 2011.

[6] D. J. Barasara, J. C. Prajapati, and A. M. Dethalia, "Multifrequency fractal antenna," International Journal of Scientific \& Engineering Research, vol. 3, no. 7, 2012.

[7] S. Niranchanan and D. Budimir, "Novel folded SRR-loaded waveguide bandpass filters," in IEEE Antennas and Propagation
Society International Symposium, pp. 1-4, San Diego, Calif, USA, 2008.

[8] A. Ishimaru, S. Jaruwatanadilok, and Y. Kuga, "Generalized surface plasmon resonance sensors using metamaterials and negative index materials," Progress in Electromagnetics Research, vol. 51, pp. 139-152, 2005.

[9] A. V. Kabashin, P. Evans, S. Pastkovsky et al., "Plasmonic nanorod metamaterials for biosensing," Nature Materials, vol. 8, no. 11, pp. 867-871, 2009.

[10] A. Alù and N. Engheta, "Dielectric sensing in $\varepsilon$-near-zero narrow waveguide channels," Physical Review B-Condensed Matter and Materials Physics, vol. 78, no. 4, Article ID 045102, 2008.

[11] E. Ekmekci and G. Turhan-Sayan, "Multi-functional metamaterial sensor based on a broad-side coupled SRR topology with a multi-layer substrate," Applied Physics A: Materials Science and Processing, vol. 110, no. 1, pp. 189-197, 2013.

[12] L. La Spada, F. Bilotti, and L. Vegni, "Metamaterial-based sensor design working in infrared frequency range," Progress in Electromagnetics Research B, vol. 34, pp. 205-223, 2011.

[13] C. Drexler, T. V. Shishkanova, C. Lange et al., “Terahertz splitring metamaterials as transducers for chemical sensors based on conducting polymers: a feasibility study with sensing of acidic and basic gases using polyaniline chemosensitive layer," Microchimica Acta, vol. 181, no. 15-16, pp. 1857-1862, 2014.

[14] F. Miyamaru, K. Hattori, K. Shiraga et al., Highly Sensitive Terahertz Sensing of Glycerol-Water Mixtures with Metamaterials, Springer Science+Business Media New York, New York, NY, USA, 2013.

[15] C. Mandel, B. Kubina, M. Schüßler, and R. Jakoby, "Metamaterial-inspired passive chipless radio-frequency identification and wireless sensing," Annals of Telecommunications, vol. 68, no. 7-8, pp. 385-399, 2013.

[16] A. C. Strikwerda, H. Tao, E. A. Kadlec et al., "Metamaterial based terahertz detector," in Proceedings of the 36th International Conference on Infrared, Millimeter, and Terahertz Waves (IRMMW-THz '11), pp. 1-2, Houston, Tex, USA, October 2011.

[17] M. Puentes, C. Weiß, M. Schüßler, and R. Jakoby, "Sensor array based on split ring resonators for analysis of organic tissues," in Proceedings of the IEEE MTT-S International Microwave Symposium Digest (MTT '11), pp. 1-4, IEEE, Baltimore, Md, USA, June 2011.

[18] H.-J. Lee, J.-H. Lee, and H.-I. Jung, "A symmetric metamaterial element-based RF biosensor for rapid and label-free detection," Applied Physics Letters, vol. 99, no. 16, Article ID 163703, 2011.

[19] T. Chen, S. Li, and H. Sun, "Metamaterials application in sensing," Sensors, vol. 12, no. 3, pp. 2742-2765, 2012.

[20] R. Melik, E. Unal, N. K. Perkgoz, C. Puttlitz, and H. V. Demir, "Metamaterial-based wireless RF-MEMS strain sensors," in Proceedings of the 9th IEEE Sensors Conference (SENSORS '10), pp. 2173-2176, November 2010.

[21] J. D. Baena, J. Bonache, F. Martín et al., "Equivalent-circuit models for split-ring resonators and complementary splitring resonators coupled to planar transmission lines," IEEE Transactions on Microwave Theory and Techniques, vol. 53, no. 4, pp. 1451-1460, 2005.

[22] J.-G. Hyun, S. Lee, S.-D. Cho, and K.-W. Paik, "Frequency and temperature dependence of dielectric constant of epoxy/ $/ \mathrm{BaTiO}_{3}$ composite Embedded Capacitor Films (ECFs) for organic substrate," in Proceedings of the 55th Electronic Components and Technology Conference (ECTC '05), pp. 1241-1247, IEEE, June 2005. 
[23] S. Liebus, Couches minces ferroélectriques appliquées aux dispositifs microondes accordables en fréquence [Ph.D. thesis], Université de Limoges, Limoges, France, 2003.

[24] R. W. Ziolkowski, "Design, fabrication, and testing of double negative metamaterials," IEEE Transactions on Antennas and Propagation, vol. 51, no. 7, pp. 1516-1529, 2003.

[25] S. S. Mohan, "The design, modeling and optimization of on-chip inductor and transformer circuits," December 1999.

[26] M. F. Wu, F. Y. Meng, Q. Wu, J. Wu, and L. W. Li, "A compact equivalent circuit model for the SRR structure in metamaterials," in Proceedings of the Asia-Pacific Conference Proceedings; Microwave Conference Proceedings (APMC '05), vol. 1, December 2005.

[27] F. Jona and G. Shirane, Ferroelectric Crystals, Dover, New York, NY, USA, 1993. 

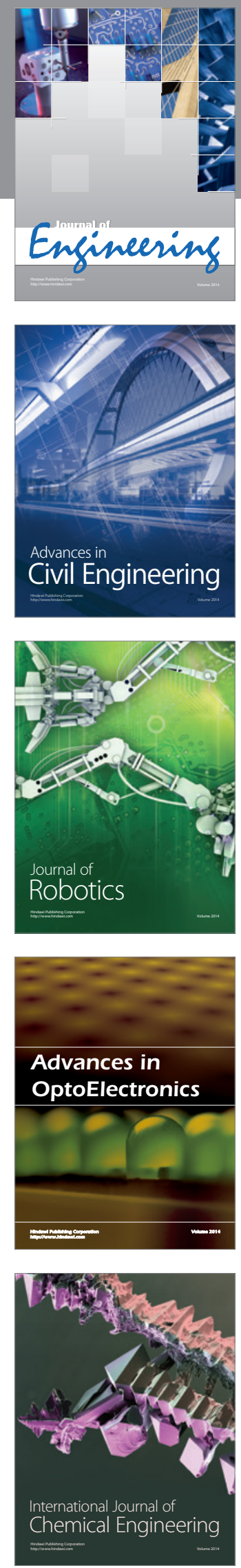

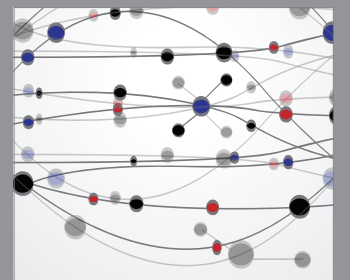

The Scientific World Journal
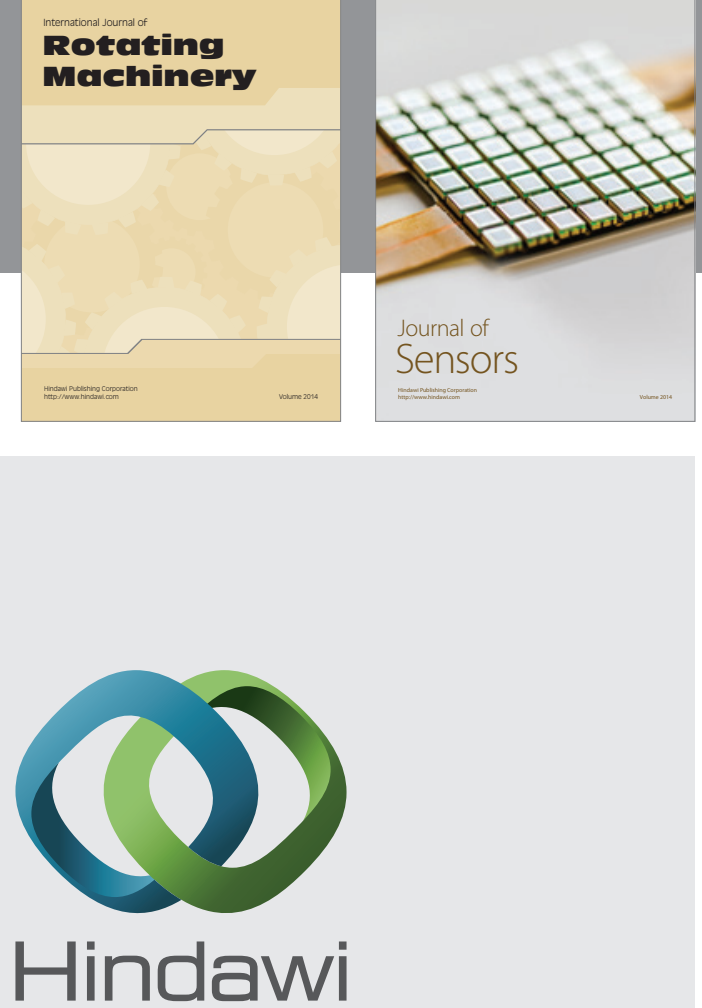

Submit your manuscripts at http://www.hindawi.com
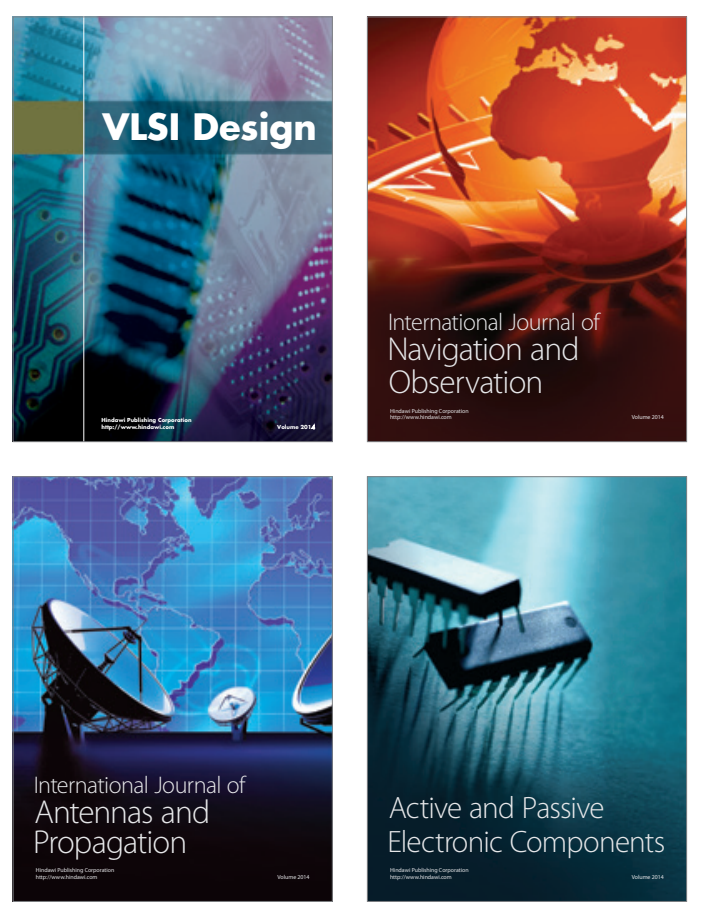
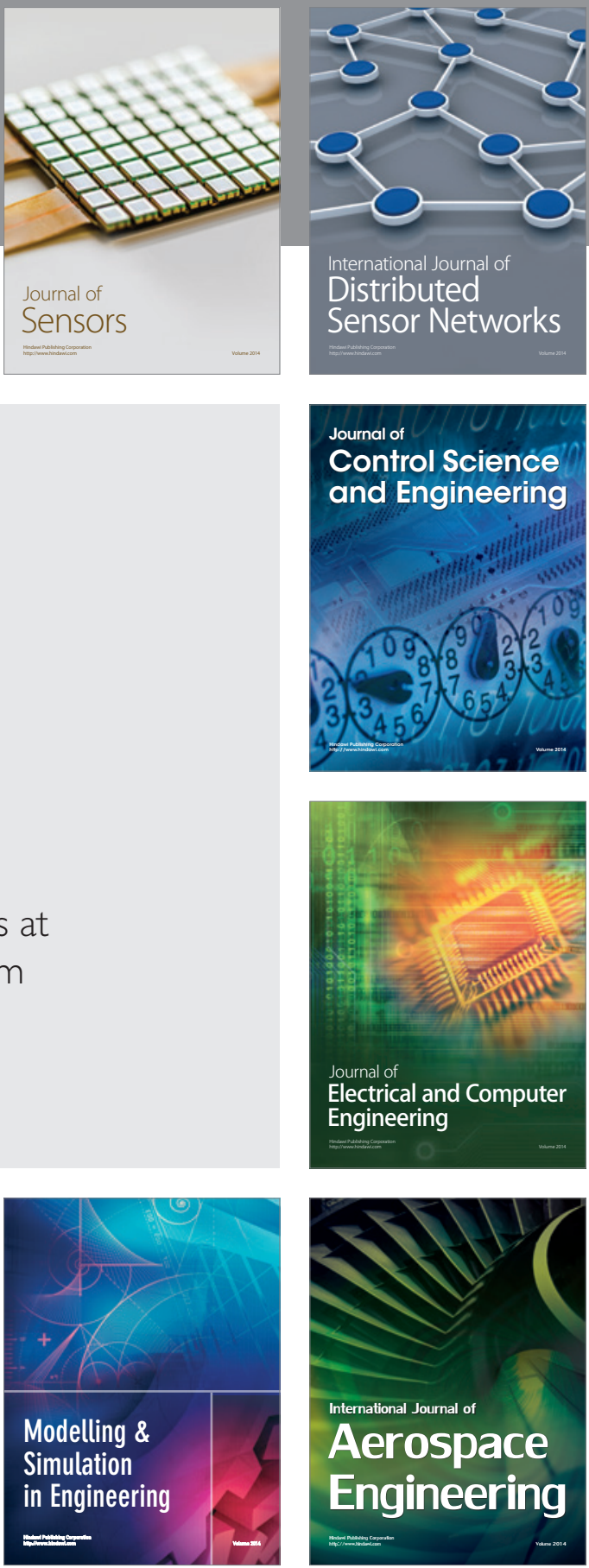

Journal of

Control Science

and Engineering
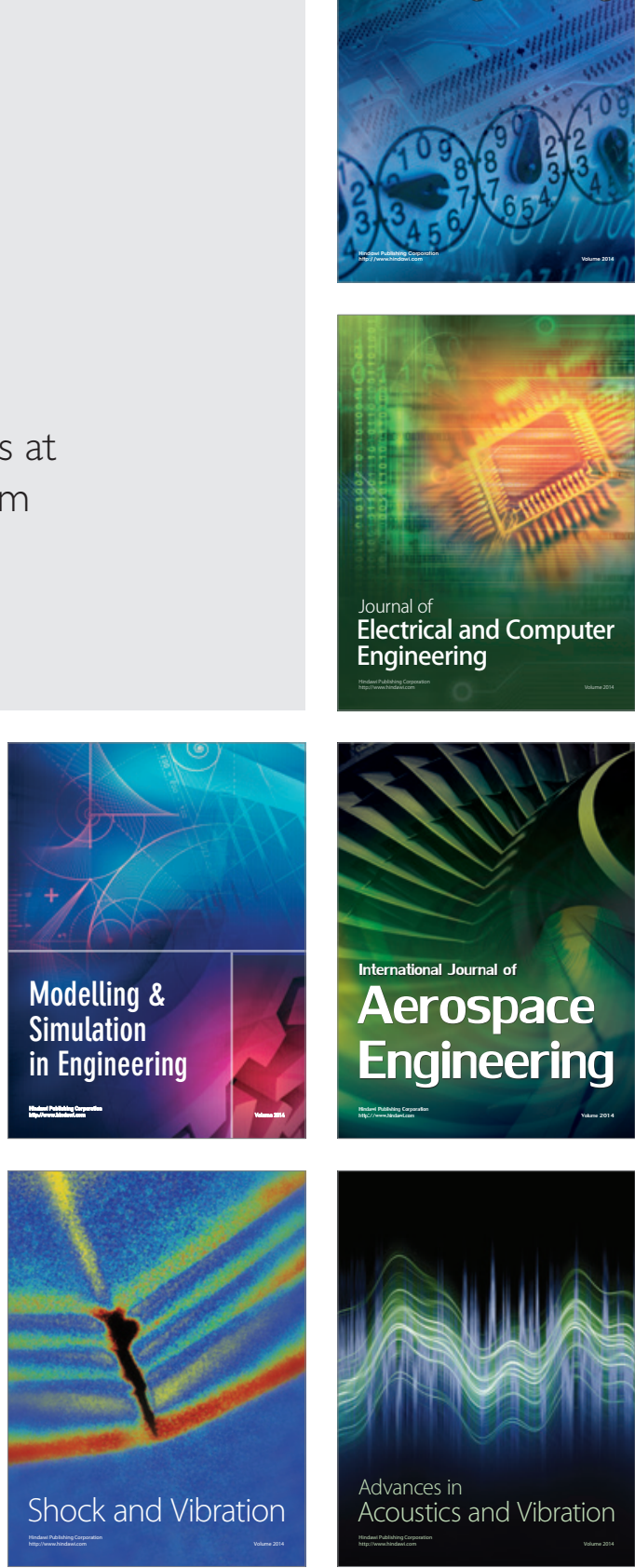